\title{
Vitamin D protects glomerular mesangial cells from high glucose-induced injury by repressing JAK/STAT signaling
}

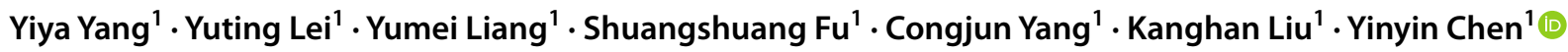

Received: 23 February 2020 / Accepted: 2 December 2020 / Published online: 3 May 2021

(c) The Author(s) 2021

\begin{abstract}
Aim High glucose (HG) induces the production of transforming growth factor (TGF)- $\beta$ and reactive oxygen species, which further activates JAK/STAT signaling and promotes the synthesis of matrix proteins, contributes to the pathophysiological processes of diabetic nephropathy. This study aims to investigate the protection role of vitamin D (VD) in the kidney in high glucose condition.

Methods Rat glomerular mesangial cells were cultured in high glucose medium, with or without VD or VD receptor (VDR) siRNAs treatment. The levels of TGF- $\beta$ and fibronectin were detected by qRT-PCR, immunoblotting and enzyme-linked immunosorbent assay (ELISA). The levels of phosphorylated JAK2, STAT1 and STAT3, and JAK/STAT signaling downstream genes were examined by immunoblotting and qRT-PCR.

Results In rat glomerular mesangial cells, VD treatment can repress the tyrosine phosphorylation of JAK2, STAT1 and STAT3. VD inhibited TGF- $\beta$ and fibronectin expression which was rescued by vitamin d receptor (VDR) siRNA and STATs inhibitor perficitinib. The JAK/STAT signaling downstream protein coding genes including SOCS1, SOCS3 and type IV collagen were repressed by VD. Meanwhile, the expression of non-coding RNAs such as miR-181a, miR-181b, was repressed by VD, and the expression of miR-34a and Let-7b was upregulated by VD.

Conclusion Vitamin D (VD) treatment inhibits the function of HG on fibronectin production through regulating JAK/STAT pathway. These results provide direct evidences that VD protects glomerular mesangial cells from high glucose-induced injury through repressing JAK/STAT signaling, which has the potential for clinical DN treatment.
\end{abstract}

Keywords Reactive oxygen species $\cdot$ High glucose $\cdot$ Vitamin D $\cdot$ JAK/STAT $\cdot$ Glumerular cell

\section{Introduction}

Diabetic nephropathy (DN) is a common complication usually found in patients with diabetes mellitus (DM), which is accounting for chronic renal failure [1]. Although it is reported that abnormal renal hemodynamics, hyperglycemia-induced metabolic disorders, and the imbalance of vasoactive substances contribute to the development of DN, the underlying mechanisms for diabetic nephropathy are still not

Yinyin Chen

chenchenyinyin1212@163.com

1 Department of Nephrology and Laboratory of Kidney Disease, Changsha Clinical Research Center for Kidney DiseaseHunan Clinical Research Center for Chronic Kidney Disease, Hunan Provincial People's Hospital, Hunan Normal University, 61\#, Jiefang West Road, Changsha 410005, Hunan Province, People's Republic of China totally unveiled $[2,3]$. Evidences indicate that the production of reactive oxygen species (ROS) is increased during hyperglycemia and the oxidative stress play a significant role during the development and progression of DN $[4,5]$. ROS can stimulate the activity of the known STAT kinases JAK2 and TYK2, and the activation of the JAK/STAT signaling cascade further stimulates excessive proliferation and growth of glomerular mesangial cells, contributing to diabetic nephropathy $[6,7]$.

Numerous evidences indicate that hyperglycemia can also induce an increase of transforming growth factor- $\beta 1$ (TGF- $\beta$ ) expression in cultured mesangial cells and diabetic mouse models [8-10]. Increased renal production of TGF- $\beta$ was also observed in patients with diabetes and DN [11, 12]. TGF- $\beta 1$ is a multifunctional cytokine the overexpression of which will enhance the synthesis and cross-linking of extracellular matrix (ECM) [13, 14]. Researchers found that neutralization of TGF- $\beta$ prevented glomerulosclerosis 
and interstitial fibrosis, and reduced the expression of many ECM genes including fibronectin and type IV collagen in diabetic mice $[15,16]$.

Fibronectins are a group of large glycoproteins which are encoded by a single gene FN1. Fibronectins are key components of extracellular matrix which promote cell-cell interactions. A significant increase of fibronectin was reported in most types of glomerulopathy including DN, which contributes to aberrant thickening of glomerular basement membranes and excessively amassing of mesangial matrices [17-19].

Vitamin D has calciotropic and pleiotropic effects mediated through vitamin D receptor (VDR) expressed in different organs including kidney [20]. The VD deficiency is associated with increased urinary albumin excretion, as well as an increase in the prevalence of cardiovascular disease and mortality in patients with chronic kidney disease in the general population [21, 22]. Vitamin D was reported can inhibit JAK/STAT signaling in hepatocytes and T cells [23, 24]. Growing evidence indicates that VD deficiency could be a contributing factor in the development of both type 1 and type 2 diabetes. Evidences indicate that VD treatment improves glucose tolerance and insulin resistance by regulating insulin secretion [25-27]. However, whether VD protects kidney from HG-induced ROS injury, and what is the underlying mechanism are unclear. In the present study, we confirmed and examined the function of VD on the JAK/ STAT signaling, TGF- $\beta$ production and the expression of fibronectin in glomerular mesangial cells.

\section{Materials and methods}

\section{Cell culture}

Glomerular mesangial cells were isolated from rat glomeruli as previously described [28]. Briefly, glomeruli were obtained from male Sprague-Dawley rats and incubated with 250 units/mL collagenase I (SCR103, Sigma-Aldrich, St. Louis, MO, USA) for $30 \mathrm{~min}$ at $37{ }^{\circ} \mathrm{C}$. Glomerular mesangial cells were plated on tissue culture dishes in DMEM (11966025, Gibco, Thermo Fisher Scientific, Inc., Waltham, MA, USA) either with normal concentration glucose (NG) (A2494001, Thermo Fisher Scientific, Inc., Waltham, MA, USA) $(5.5 \mathrm{mmol} / \mathrm{L})$ or with high concentration glucose $(\mathrm{HG})$ ( $25 \mathrm{mmol} / \mathrm{L}$ ) supplemented with $10 \%$ fetal bovine serum (26140, lot 1566368, Gibco, Thermo Fisher Scientific, Inc, Waltham, MA, USA), $100 \mathrm{IU} / \mathrm{ml}$ penicillin and $10 \mathrm{mg} / \mathrm{mL}$ streptomycin (15140122, Gibco, Thermo Fisher Scientific, Inc., Waltham, MA, USA). All cells were maintained at $37{ }^{\circ} \mathrm{C}$ under an atmosphere of $5 \% \mathrm{CO} 2$.

\section{ROS detection}

(a) The total intracellular superoxide and hydroxyl radical in cells was detected using Cellular Reactive Oxygen Species Detection Assay Kit (ab186027, Abcam, Cambridge, UK), following the manufacturer's instructions. Briefly, cells were seeded into 96 -well plate with $2 \times 10^{4}$ cells per well. Add $100 \mu \mathrm{L} /$ well of ROS Red Working Solution into the plate. Incubate cell plate in a $37{ }^{\circ} \mathrm{C} / 5 \% \mathrm{CO}_{2}$ incubator for $1 \mathrm{~h}$, and then detect the fluorescence signal by microplate reader.

(b) Intracellular hydrogen peroxide detection. Cells were plated into 12-well plates with different treatments. Each well was loaded with $30 \mu \mathrm{m}$ of the $\mathrm{H} 2 \mathrm{O} 2$-sensitive fluorescent probe 2',7'-dichlorofluorescein diacetate (DCFDA; Molecular Probes, Leiden, The Netherlands) and then the plate was plated at $37{ }^{\circ} \mathrm{C}$ for $4 \mathrm{~h}$. The cells were washed with PBS for three times and then the intracellular fluorescence was quantified using flow cytometry.

(c) The level of superoxide anion was determined by ethidium fluorescence. Briefly, cells were seeded into 12-well plates wit different treatments. The cells were incubated with $1 \mathrm{mM}$ hydroethidine at $37^{\circ} \mathrm{C}$ for $5 \mathrm{~min}$ and then washed three times by PBS. The cells were then resuspended and subjected to flow cytometry analysis.

\section{Cell transfection}

Small interfering RNA (siRNA) targeting VDR was transfected into cells using Lipofectamine 2000 Transfection Reagent (Thermo Fisher Scientific, Inc., Waltham, MA, USA), following the manufacturer's instructions. siVDR sequence: 5'- rCrCrArArGrCrUrArUrCrUrGrArArGrArArCrArArCrArGCA-3' and 5'- rUrGrCrUrGrUrUrGrUrUrCrUrUrCrArGrArUrArGrCrUrUrGrGrGrC-3'.

\section{Enzyme-linked immunosorbent assay (ELISA)}

The level of secreted TGF- $\beta$ and fibronectin proteins under the different experimental conditions were quantified using commercial ELISA kits following the manufacturer's instructions. Rat TGF beta 1 ELISA Kit (ab119558, Abcam, Cambridge, UK) (sensitivity of the kit: higher than $7.8 \mathrm{pg} /$ $\mathrm{mL}$ ). Rat Fibronectin ELISA Kit (ab108850, Abcam, Cambridge, UK) (sensitivity of the kit: higher than $75 \mathrm{pg} / \mathrm{mL}$ ).

\section{Immunoblotting}

Protein samples were quantified using a Pierce BCA Protein Assay Kit (Thermo Fisher Scientific, Inc., Waltham, MA, USA), according to the manufacturer's protocols. Protein samples were heated with SDS sample buffer $(0.25 \mathrm{M}$ 
Tris-HCl, pH 6.8, 0.5 M DTT, 10\% SDS, 50\% glycerol and $0.5 \%$ bromophenol blue) at $95{ }^{\circ} \mathrm{C}$ for $5 \mathrm{~min}$, a total of $30 \mu \mathrm{g}$ were loaded per lane and then separated on 15\% SDS-PAGE and electroblotted onto polyvinylidene fluoride membranes (GE10600023, GE Healthcare Life Sciences, Logan, UT, USA). Subsequently, the membranes were incubated with one of the primary rabbit polyclonal antibodies at a dilution of 1:1,000 (Abcam) or anti- $\beta$-actin rabbit monoclonal antibody at a dilution of 1:5,000 (Abcam) overnight at $4{ }^{\circ} \mathrm{C}$. Immunoreactive bands were detected by incubation with horseradish peroxidase-conjugated goat anti-rabbit (Abcam) at a dilution of 1:5,000 for $2 \mathrm{~h}$ at room temperature. Detection by chemiluminescence was performed using a Pierce enhanced chemiluminescence kit (Thermo Fisher Scientific, Inc., Waltham, MA, USA).

\section{Antibody information}

Rabbit anti-fibronectin polyclonal antibody (ab2413, Lot. No. GR3261228-3, Abcam, Cambridge, UK); rabbit antiphospho-JAK2 monoclonal antibody (ab32101, Lot. No. GR321849-8, Abcam, Cambridge, UK); rabbit anti-JAK2 monoclonal antibody(ab108596, Lot. No. GR261730-3, Abcam, Cambridge, UK); rabbit anti-VDR monoclonal antibody (ab109234, Lot. No. GR223547-2, Abcam, Cambridge, UK); rabbit anti-STAT1 monoclonal antibody(ab234400, Lot. No. GR323412-8, Abcam, Cambridge, UK); rabbit monoclonal anti-STAT1 (phosphor-Y701) antibody (ab109457, Lot. No. GR311243-6, Abcam, Cambridge, UK); rabbit anti-STAT3 monoclonal antibody (ab68153, Lot No. 32103-2, Abcam, Cambridge, UK), rabbit anti-STAT3 (phosphor-Y705) monoclonal antibody (ab76315, Lot. No. 312232-2, Abcam, Cambridge, UK), rabbit anti- $\beta$-actin polyclonal antibody (ab8227, Lot. No. 213113-8, Abcam, Cambridge, UK).

\section{RNA extraction and RT-qPCR}

Total RNA from cells was extracted using a miRNeasy Mini kit (217004, Qiagen, Hilden, Germany) following the manufacturer's instructions. Only samples with the appropriate absorbance measurements (A260/A280 of 2.0, and A260/A230 of 1.9-2.2) were considered for inclusion in the present study. RT-qPCR was applied to detect the relative expression levels of selected mRNAs and miRNAs. The reaction conditions were as follows: $95{ }^{\circ} \mathrm{C}$ for $10 \mathrm{~min}$, followed by 40 cycles of $95^{\circ} \mathrm{C}$ for $15 \mathrm{~s}$ and $60{ }^{\circ} \mathrm{C}$ for $1 \mathrm{~min}$. To compare the miRNA levels between different samples, the $2-\Delta \Delta \mathrm{Cq}$ method was used. The level of GAPDH was used as loading control for mRNAs and U6 snRNA was used for miRNAs normalization. A total of three triplicate experiments were performed in each case.

\section{Statistical analysis}

Statistical analysis was performed using SPSS software version 19.0 (IBM Corp., Armonk, NY, USA) and data are presented as the mean \pm standard. The data were analyzed by Shapiro-Wilk test to examine whether the data are normal distributed. Results were then analyzed using one-way ANOVA Tukey's honestly significant difference (HSD) post hoc test. $P<0.05$ was considered to indicate a statistically significant difference.

\section{Results}

To investigate the mechanisms of diabetic nephropathy, we compared the total ROS level in glomerular mesangial cells treated with normal and high glucose. As shown in Fig. 1a, high glucose significantly alleviated the ROS level in glomerular mesangial cells, which was partially reduced by vitamin D. Meanwhile, when VDR was knocked down by siRNA, the effect of VD on ROS repression was partially inhibited. These results indicated that VD/VDR signaling play protective roles in glomerular mesangial cells in high glucose environment. Subsequently, the intracellular hydrogen peroxide and superoxide anion were detected by flow cytometry separately to investigate which ROS subtype is VD/VDR signaling is related. As shown in Fig.S1, vitamin $\mathrm{D}$ treatment reduced the level of both hydrogen peroxide and superoxide in glomerular mesangial cells. When VDR was knocked down, the protective role of vitamin D is reduced, indicating that VD/VDR signaling protected glomerular mesangial cells from the injury of high glucose-induced hydrogen peroxide and superoxide.

Accumulation of extracellular matrix (ECM) proteins, including fibronectin, is one of the main causes of glomerulosclerosis in diabetic nephropathy. To understand the roles of $\mathrm{HG}$ on ECM accumulation, the mRNA level expression of fibronectin in were examined by qRT-PCR. As shown in Fig. 1b, the fibronectin expression in mesangial cells was significantly increased by HG. When the cells were treated by $\mathrm{VD}$, the effect of $\mathrm{HG}$ on fibronectin elevation was almost totally repressed. Meanwhile, in the VDR knockdown cells, the function of VD was partially restored. Similar results were observed in the protein level of fibronectin (Fig. 1c). The fibronectin protein level was increased by HG, which was repressed by VD treatment. When VDR was knocked down by siRNA, the fibronectin protein level was similar as the cells treated by HG only.

Since HG-induced ROS can activate JAK/STAT signaling, which further upregulate fibronectin expression [28, 29], the levels of JAK2 and p-JAK2 were also examined by immunoblotting. As shown in Fig. 1c, increased p-JAK2 level was observed in cells treated by HG. VD treatment 
A

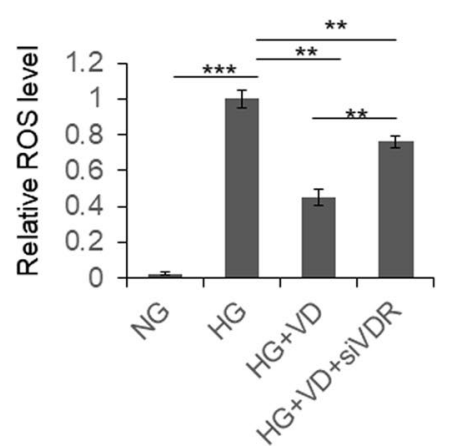

B

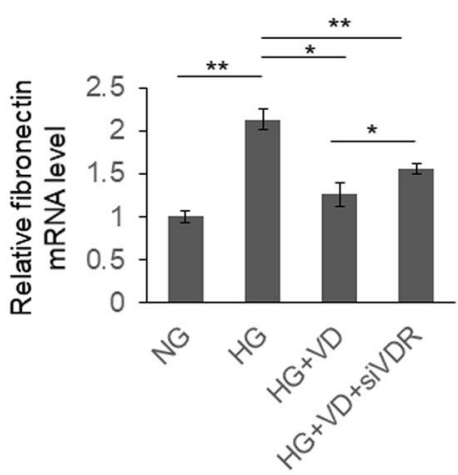

C

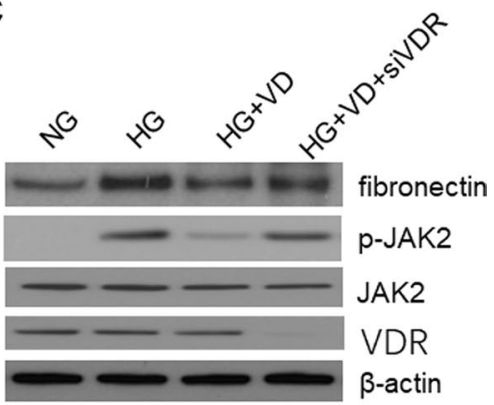

Fig. 1 VD repressed ROS and fibronectin production induced by HG. Rat glomerular mesangial cells were cultured under different conditions with or without siVDR transfection for $48 \mathrm{~h}$, and then subjected to intracellular ROS detection (a), or subjected to qRT-PCR (b) or

repressed p-JAK2 level significantly, which was restored by VDR siRNA. These results indicated that HG upregulated fibronectin expression through increasing ROS level and activating JAK/STAT signaling, which can be repressed by VD treatment.

Subsequently, the level of pJAK2, pSTAT1 and pSTAT3 in glomerular mesangial cells was examined by immunoblotting to confirm the repressive role of vitamin D. As shown in Fig. 2, under NG conditions, there is no tyrosine phosphorylation of JAK2 detected. When glomerular mesangial cells were cultured in HG conditions, p-JAK2 was significantly increased when compared with NG conditions, at both 24 and $48 \mathrm{~h}$, indicating that $\mathrm{HG}$ promotes a immunoblotting (c) to examine the expression of fibronectin. Results were analyzed by one-way ANNOVA and $p<0.05$ was considered significant. ${ }^{* *} p<<0.001,{ }^{* *} p<0.0,{ }^{*} p<0.05$

constitutive activation of JAK2. Meanwhile, VD treatment partially inhibited the HG-induced JAK2 tyrosine phosphorylation at 24 and $48 \mathrm{~h}$. When VDR was knocked down, the inhibition of VD on JAKs phosphorylation was restored.

Similar results were observed in the p-STAT1 and p-STAT3 levels. As shown in Fig. 2, HG induced a significant increased level of phosphorylated STAT1 and STAT3 compared with NG conditions at both 24 and $48 \mathrm{~h}$. Meanwhile, VD repressed the phosphorylated STAT1 and STAT3 level, which was blocked by siVDR. These results indicating that the HG promotes a constitutive activation of JAK/STAT signaling in the absence of growth factors, which can be inhibited by VD.

\section{Normal glucose}

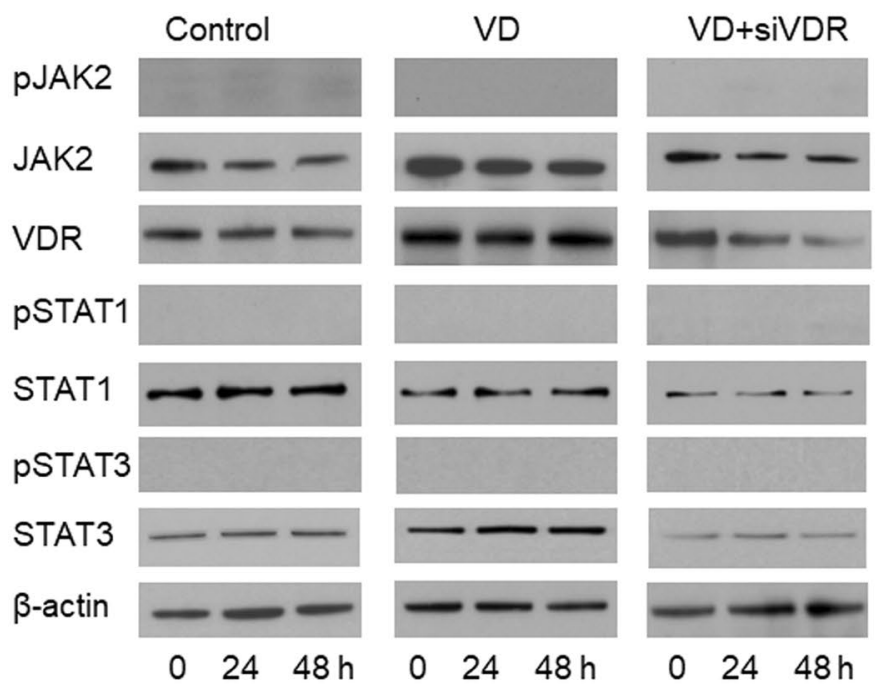

\section{High glucose}

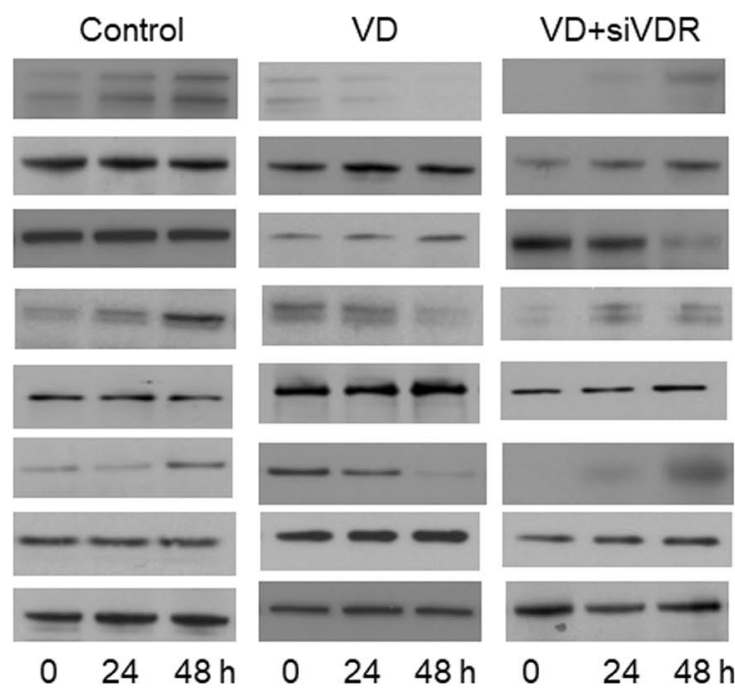

Fig. 2 VD inhibited tyrosine phosphorylation of JAK2, STAT1 and STAT3 which was induced by HG. Rat glomerular mesangial cells were cultured under different conditions with or without siVDR transfection for 24 or $48 \mathrm{~h}$, and then subjected to immunoblotting 
High glucose was found to induce TGF- $\beta$ expression, leading to production of extracellular matrix proteins [30]. In systemic sclerosis, VDR was found regulates TGF- $\beta$ Signaling [31]. To examine whether VD regulate TGF- $\beta$ Signaling in kidney, we detected the secreted TGF- $\beta$ and fibronectin levels in the medium by ELISA. As shown in Fig. $3 a, b$, TGF- $\beta$ and fibronection levels in the medium were increased significantly in the HG conditions and VD treatment partially repressed production of TGF- $\beta$ and fibronectin. Meanwhile, siRNA targeting VDR and STAT inhibitor-peficitinib can both partially restored the function of VD. Similar results were observed in the cells. As shown in Fig. 3c, HG treatment upregulated intracellular TGF- $\beta$ and fibronectin, which was repressed by VD. Si-VDR and peficitinib can both partially restored the function of VD. These results indicated that HG-induced JAK/STAT activation plays an important role in the HG-induced TGF- $\beta$ synthesis and fibronectin production.

To further identify the effect of HG on JAK/STAT activation, we examined the expression of JAK/STAT signaling pathway downstream genes, including SOCS1, SOCS3 and type IV collagen. As shown in Fig. 4a, the expression of these three genes were up-regulated under HG condition, and repressed by VD. Meanwhile, many miRNAs were reported dysregulated in patients with $\mathrm{DN}$, but the reason was not well understood [32]. JAK/STAT signaling also participates miRNAs expression regulation. So we detected four reported JAK/STAT downstream miRNAs expression in glomerular mesangial cells cultured under different conditions. As shown in Fig. 4b, the level of miR-181a and miR-181b was increased under HG condition, which was partially restored by VD. The expression of miR-34a and Let-7b was repressed under HG condition, which partially restored by VD.

\section{Discussion}

It is identified that ROS is a common denominator for the causation of renal injury in patients with diabetes. So, ROS has the potential to be a novel target to ameliorate reno-vascular complications of diabetes. ROS can stimulate the activity of STAT kinases and the activation of the JAK/STAT signaling cascade further stimulates excessive proliferation and growth of glomerular mesangial cells, and the matrix proteins expression, contributing to diabetic nephropathy $[6$, 7]. In the present study, we identified that VD treatment can reduce the amount of HG-induced ROS for the first time, which provides a candidate method for DN treatment.

MiR-181a and miR-181b were reported upregulated by JAK/STAT pathway in breast cancer cells. miR-34a and Let-7b were reported repressed by STAT3 signaling in colon cancer and breast cancer cells [33-36]. However, the relationship between these miRNAs and JAK/STAT signaling is still unknown in glomerular mesangial cells. In this study, we confirmed that HG-induced JAK/STAT activation upregulated the level of miR-181a, miR-181b and repressed the expression of miR-34a and Let-7b, which construct the link between JAK/STAT signaling and these miRNAs in glomerular mesangial cells for the first time.

VD has calciotropic and pleiotropic effects mediated through VDR expressed in kidneys, including podocytes, intestine, bones, parathyroid glands, pancreatic beta cells, monocytes, and T-cells [37]. It is reported that VD can inhibit JAK/STAT signaling in hepatocytes and T cells [23, 24]. However, the relationship between VD and JAK/STAT signaling during DN is still unknown. In this study, we confirmed that VD inhibited HG-induced JAK/STAT signaling activation which provided a candidate choice for clinical DN treatment.
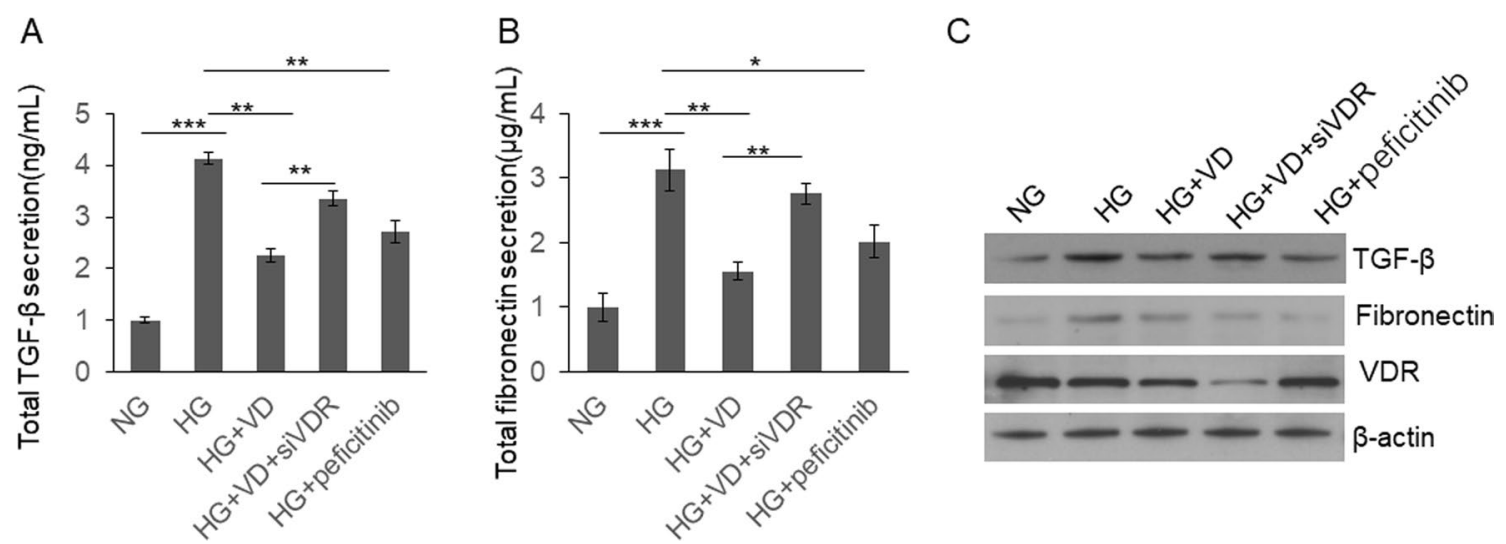

Fig. 3 VD inhibited TGF- $\beta$ and fibronectin secretion by repressing JAK/STAT signaling. Rat glomerular mesangial cells were cultured under different conditions with or without siVDR transfection or peficitinib treatment for $48 \mathrm{~h}$. The cell culture mediums were sub- jected to ELISA assay to determine secreted TGF- $\beta$ and fibronectin level. The cells were subjected to immunoblotting. Results were analyzed by one-way ANNOVA and $p<0.05$ was considered significant. $* * * p<0.001, * * p<0.0, * p<0.05$ 

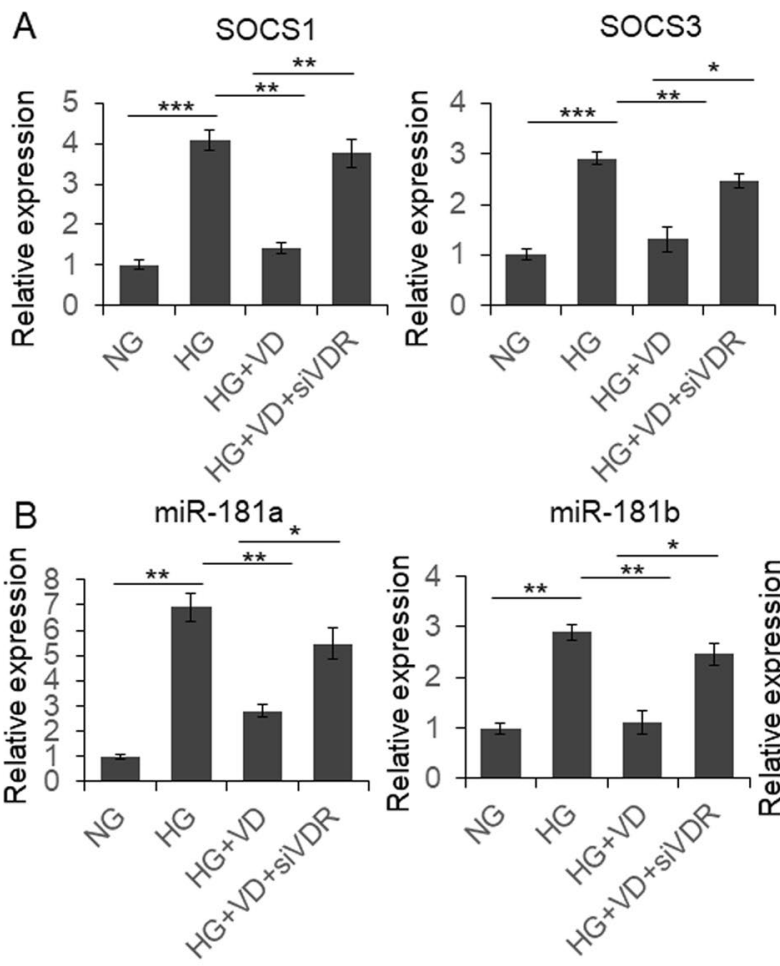

Fig. 4 VD regulated the expression of JAK/STAT downstream protein coding genes and miRNAs. Rat glomerular mesangial cells were cultured under different conditions with or without siVDR transfec-
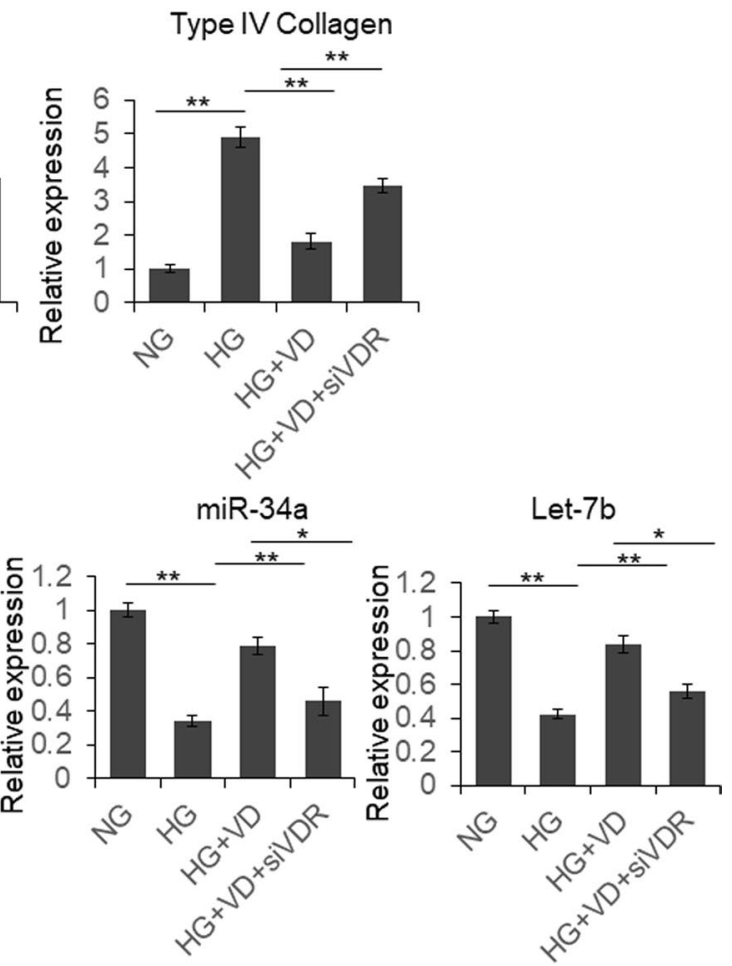

tion for $48 \mathrm{~h}$, and then subjected to qRT-PCR. Results were analyzed by one-way ANNOVA and $p<0.05$ was considered significant. $* * * p<0.001, * * p<0.0, * p<0.05$

need to obtain permission directly from the copyright holder. To view a copy of this licence, visit http://creativecommons.org/licenses/by/4.0/.

\section{References}

1. Paneni F, Beckman JA, Creager MA, Cosentino F (2013) Diabetes and vascular disease: pathophysiology, clinical consequences, and medical therapy: part I. Eur Heart J 34(31):2436-2443. https:// doi.org/10.1093/eurheartj/eht149

2. Gall MA, Rossing P, Skott P, Damsbo P, Vaag A, Bech K et al (1991) Prevalence of micro- and macroalbuminuria, arterial hypertension, retinopathy and large vessel disease in European type 2 (non-insulin-dependent) diabetic patients. Diabetologia 34(9):655-661. https://doi.org/10.1007/bf00400995

3. Remuzzi G, Benigni A, Remuzzi A (2006) Mechanisms of progression and regression of renal lesions of chronic nephropathies and diabetes. J Clin Investig 116(2):288-296. https://doi. org/10.1172/JCI27699

4. Baynes JW, Thorpe SR (1999) Role of oxidative stress in diabetic complications: a new perspective on an old paradigm. Diabetes 48(1):1-9. https://doi.org/10.2337/diabetes.48.1.1

5. Ceriello A, Morocutti A, Mercuri F, Quagliaro L, Moro M, Damante G et al (2000) Defective intracellular antioxidant enzyme production in type 1 diabetic patients with nephropathy. Diabetes 49(12):2170-2177. https://doi.org/10.2337/diabe tes.49.12.2170

6. Simon AR, Rai U, Fanburg BL, Cochran BH (1998) Activation of the JAK-STAT pathway by reactive oxygen species. Am J Physiol 
275(6):C1640-C1652. https://doi.org/10.1152/ajpcell.1998.275.6. $\mathrm{C} 1640$

7. Marrero MB, Banes-Berceli AK, Stern DM, Eaton DC (2006) Role of the JAK/STAT signaling pathway in diabetic nephropathy. Am J Physiol Renal Physiol 290(4):F762-F768. https://doi.org/ 10.1152/ajprenal.00181.2005

8. Park IS, Kiyomoto H, Abboud SL, Abboud HE (1997) Expression of transforming growth factor-beta and type IV collagen in early streptozotocin-induced diabetes. Diabetes 46(3):473-480. https:// doi.org/10.2337/diab.46.3.473

9. Wahab NA, Harper K, Mason RM (1996) Expression of extracellular matrix molecules in human mesangial cells in response to prolonged hyperglycaemia. Biochem J 316(Pt 3):985-992. https:// doi.org/10.1042/bj3160985

10. Sharma K, Ziyadeh FN (1995) Hyperglycemia and diabetic kidney disease. The case for transforming growth factor-beta as a key mediator. Diabetes 44(10):1139-1146. https://doi.org/10.2337/ diab.44.10.1139

11. Iwano M, Kubo A, Nishino T, Sato H, Nishioka H, Akai Y et al (1996) Quantification of glomerular TGF-beta 1 mRNA in patients with diabetes mellitus. Kidney Int 49(4):1120-1126. https://doi.org/10.1038/ki.1996.162

12. Yamamoto T, Noble NA, Cohen AH, Nast CC, Hishida A, Gold LI et al (1996) Expression of transforming growth factor-beta isoforms in human glomerular diseases. Kidney Int 49(2):461-469. https://doi.org/10.1038/ki.1996.65

13. Roberts AB, Heine UI, Flanders KC, Sporn MB (1990) Transforming growth factor-beta. Major role in regulation of extracellular matrix. Ann N Y Acad Sci 580:225-232. https://doi.org/10. 1111/j.1749-6632.1990.tb17931.x

14. Boak AM, Roy R, Berk J, Taylor L, Polgar P, Goldstein RH et al (1994) Regulation of lysyl oxidase expression in lung fibroblasts by transforming growth factor-beta 1 and prostaglandin E2. Am J Respir Cell Mol Biol 11(6):751-755. https://doi.org/10.1165/ ajrcmb.11.6.7946403

15. Sharma K, Jin Y, Guo J, Ziyadeh FN (1996) Neutralization of TGF-beta by anti-TGF-beta antibody attenuates kidney hypertrophy and the enhanced extracellular matrix gene expression in STZ-induced diabetic mice. Diabetes 45(4):522-530. https://doi. org/10.2337/diab.45.4.522

16. Ziyadeh FN, Hoffman BB, Han DC, Iglesias-De La Cruz MC, Hong SW, Isono M et al (2000) Long-term prevention of renal insufficiency, excess matrix gene expression, and glomerular mesangial matrix expansion by treatment with monoclonal antitransforming growth factor-beta antibody in $\mathrm{db} / \mathrm{db}$ diabetic mice. Proc Natl Acad Sci USA 97(14):8015-8020. https://doi.org/10. 1073/pnas. 120055097

17. Dixon AJ, Burns J, Dunnill MS, McGee JO (1980) Distribution of fibronectin in normal and diseased human kidneys. J Clin Pathol 33(11):1021-1028. https://doi.org/10.1136/jcp.33.11.1021

18. Hu C, Sun L, Xiao L, Han Y, Fu X, Xiong X et al (2015) Insights into the mechanisms involved in the expression and regulation of extracellular matrix proteins in diabetic nephropathy. Curr Med Chem 22(24):2858-2870. https://doi.org/10.2174/0929867322 666150625095407

19. Schena FP, Gesualdo L (2005) Pathogenetic mechanisms of diabetic nephropathy. J Am Soc Nephrol 16(Suppl 1):S30-S33. https://doi.org/10.1681/asn.2004110970

20. Zehnder D, Bland R, Williams MC, McNinch RW, Howie AJ, Stewart PM et al (2001) Extrarenal expression of 25-hydroxyvitamin d(3)-1 alpha-hydroxylase. J Clin Endocrinol Metab 86(2):888-894. https://doi.org/10.1210/jcem.86.2.7220

21. Mehrotra R, Kermah DA, Salusky IB, Wolf MS, Thadhani RI, Chiu YW et al (2009) Chronic kidney disease, hypovitaminosis D, and mortality in the United States. Kidney Int 76(9):977-983. https://doi.org/10.1038/ki.2009.288
22. de Boer IH, Ioannou GN, Kestenbaum B, Brunzell JD, Weiss NS (2007) 25-Hydroxyvitamin D levels and albuminuria in the Third National Health and Nutrition Examination Survey (NHANES III). Am J Kidney Dis 50(1):69-77. https://doi.org/10.1053/j.ajkd. 2007.04.015

23. Lange CM, Gouttenoire J, Duong FH, Morikawa K, Heim MH, Moradpour D (2014) Vitamin D receptor and Jak-STAT signaling crosstalk results in calcitriol-mediated increase of hepatocellular response to IFN-alpha. J Immunol 192(12):6037-6044. https:// doi.org/10.4049/jimmunol.1302296

24. Olson KC, Kulling PM, Olson TL, Tan SF, Rainbow RJ, Feith DJ et al (2017) Vitamin D decreases STAT phosphorylation and inflammatory cytokine output in T-LGL leukemia. Cancer Biol Ther 18(5):290-303. https://doi.org/10.1080/15384047.2016. 1235669

25. Parekh D, Sarathi V, Shivane VK, Bandgar TR, Menon PS, Shah NS (2010) Pilot study to evaluate the effect of short-term improvement in vitamin $\mathrm{D}$ status on glucose tolerance in patients with type 2 diabetes mellitus. Endocr Pract 16(4):600-608. https://doi.org/ 10.4158/EP09300.OR

26. von Hurst PR, Stonehouse W, Coad J (2010) Vitamin D supplementation reduces insulin resistance in South Asian women living in New Zealand who are insulin resistant and vitamin D deficient - a randomised, placebo-controlled trial. Br J Nutr 103(4):549-555. https://doi.org/10.1017/S0007114509992017

27. Bourlon PM, Billaudel B, Faure-Dussert A (1999) Influence of vitamin D3 deficiency and 1,25 dihydroxyvitamin D3 on de novo insulin biosynthesis in the islets of the rat endocrine pancreas. J Endocrinol 160(1):87-95. https://doi.org/10.1677/joe.0.1600087

28. Wang X, Shaw S, Amiri F, Eaton DC, Marrero MB (2002) Inhibition of the Jak/STAT signaling pathway prevents the high glucoseinduced increase in tgf-beta and fibronectin synthesis in mesangial cells. Diabetes 51(12):3505-3509. https://doi.org/10.2337/diabe tes.51.12.3505

29. Matsui F, Meldrum KK (2012) The role of the Janus kinase family/signal transducer and activator of transcription signaling pathway in fibrotic renal disease. J Surg Res 178(1):339-345. https:// doi.org/10.1016/j.jss.2012.06.050

30. Ziyadeh FN, Sharma K, Ericksen M, Wolf G (1994) Stimulation of collagen gene expression and protein synthesis in murine mesangial cells by high glucose is mediated by autocrine activation of transforming growth factor-beta. J Clin Investig 93(2):536542. https://doi.org/10.1172/JCI117004

31. Zerr P, Vollath S, Palumbo-Zerr K, Tomcik M, Huang J, Distler A et al (2015) Vitamin D receptor regulates TGF-beta signalling in systemic sclerosis. Ann Rheum Dis 74(3):e20. https://doi.org/ 10.1136/annrheumdis-2013-204378

32. Simpson K, Wonnacott A, Fraser DJ, Bowen T (2016) MicroRNAs in diabetic nephropathy: from biomarkers to therapy. Curr DiabRep 16(3):35. https://doi.org/10.1007/s11892-016-0724-8

33. Lei K, Du W, Lin S, Yang L, Xu Y, Gao Y et al (2016) 3B, a novel photosensitizer, inhibits glycolysis and inflammation via miR-155-5p and breaks the JAK/STAT3/SOCS1 feedback loop in human breast cancer cells. Biomed Pharmacother 82:141-150. https://doi.org/10.1016/j.biopha.2016.04.049

34. Niu J, Xue A, Chi Y, Xue J, Wang W, Zhao Z et al (2016) Induction of miRNA-181a by genotoxic treatments promotes chemotherapeutic resistance and metastasis in breast cancer. Oncogene 35(10):1302-1313. https://doi.org/10.1038/onc.2015.189

35. Li H, Rokavec M, Hermeking H (2015) Soluble IL6R represents a miR-34a target: potential implications for the recently identified IL-6R/STAT3/miR-34a feed-back loop. Oncotarget 6(16):1402614032. https://doi.org/10.18632/oncotarget.4334

36. Guo L, Chen C, Shi M, Wang F, Chen X, Diao D et al (2013) Stat3coordinated Lin-28-let-7-HMGA2 and miR-200-ZEB1 circuits initiate and maintain oncostatin M-driven epithelial-mesenchymal 
transition. Oncogene 32(45):5272-5282. https://doi.org/10.1038/ onc.2012.573

37. Prabhu RA, Saraf K (2018) Vitamin D in diabetic nephropathy. J Postgrad Med 64(1):5-6. https://doi.org/10.4103/jpgm.JPGM_ 311_17
Publisher's Note Springer Nature remains neutral with regard to jurisdictional claims in published maps and institutional affiliations. 